\title{
Impact of Mental Rotation Strategy on Absolute Direction Judgments: Supplementing Conventional Measures with Eye Movement Data
}

\author{
Ronggang Zhou ${ }^{1}$ and Kan Zhang ${ }^{2}$ \\ ${ }^{1}$ Department of Industrial Engineering, Tsinghua University, Beijing 100084, China \\ ${ }^{2}$ State Key Laboratory of Brain and Cognitive Science, Institute of Psychology, Chinese \\ Academy of Sciences, Beijing 100101, China \\ zhourg@tsinghua.edu.cn, zhangk@psych.ac.cn
}

\begin{abstract}
By training participants to use map-first mental rotation as their primary strategy on absolute navigational task, this study focused on how integration of heading information (from the exocentric reference frame) with target position information (from the egocentric reference frame) affects absolute direction judgments. Comparing with previous studies, the results in this study showed (1) response was not better for north than for south, (2) response was the slowest for back position in canonical position condition, and (3) the cardinal direction advantage of right-back position was not impaired. Eye movement data supported these conclusions partially, and should be cautious to use for similar goals. These findings can be applied to navigational training and interfaces design such as electric space.
\end{abstract}

Keywords: absolute direction judgments, mental rotation strategy, eye movement, reference frame.

\section{Introduction}

People can not specify spatial location and orientation of objects relative to other objects without using, at least basically, a frame of reference. The distinction between egocentric, or body-reference frame and exocentric, or world-reference frame is used most frequently to classify spatial reference frames for the purposes of understanding human spatial navigation [1] [2] [3]. The egocentric reference frame presents spatial knowledge with respect to navigator's view using words such as front, back, left, right, up, and down; the exocentric frame defines navigational relations with respect to bird's-eye perspective in the word using environmental elements such as the landmarks, cardinal direction (e.g. north, south, east, and west), and compass coordinates [1] [2] [3]. Relative and absolute spatial relation can be presented with using a single required corresponding reference frame. However most people are familiar with using a map to make navigation decision on way finding in an unfamiliar crossing road, and in this situation direction judgments should be based on integrating information from the exocentric reference frame (the map) with egocentric frame (the navigator's forward view). In this study, we focused on navigational tasks 
like the decision of "turn to south" in the situation, and participants were trained with using mental rotation strategy and provided egocentric and exocentric reference frames to identify direction using terminology of north $(\mathrm{N})$, north-east $(\mathrm{NE})$, east $(\mathrm{E})$, south-east (SE), south (S), south-west (SW), west (W), and north-west (NW)-we called this absolute direction judgments. Among theses points, the point of N, E, S, and $\mathrm{W}$ are four main points, direction judgments using these four concepts are call cardinal direction judgments.

\subsection{Previous Studies Review}

Absolute Direction Judgments Studies. Recently absolute direction judgments have been more investigated with starting of cardinal direction judgments studies. The representative task used in these absolute direction judgments studies can be shown in Fig. 1. Participants saw a north-up map indicating the location and heading of observer, and a ground target ahead of the observer (right part of Fig.1), and the heading can be selected from $\mathrm{N}$ to $\mathrm{NW}$ in $45^{\circ}$ or $30^{\circ}$ increments. In particular heading, the ground target was provided with corresponding forward view, which showed a central building surrounded symmetrically with four lots (left part of Fig.1). When read the 3D display, participants would be deictic to the central building, so the target lot would be located in canonical position (e.g. front $(\mathrm{F})$, back (B), left (L), or right (R)) or noncanonical position (e.g. front-left (FL), front-right (FR), back-left (BL), or back-right (BR)) relative to the reference building. The task was to determine whether the target object was N, NE, E, SE, S, SW, W, and NW of the central building.

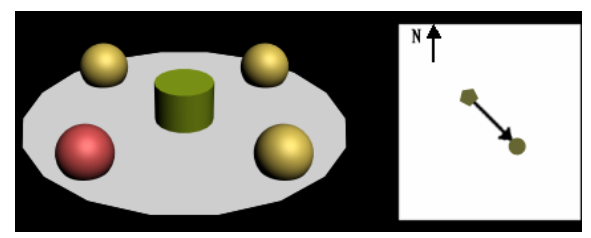

Fig. 1. Map display (exocentric reference frame) and three-dimensional display (egocentric reference frame) used in the typical absolute direction task. In the map, the heading was southeast, so the red target lot in the 3D display was north of the building. The actual displays were in color, and objects can be discriminated clearly.

The results of cardinal direction judgments studies indicated that (1) reference frame misalignment was found to slow judgment performance (slow response time and decrease accuracy), and the advantage effect of cardinal direction follow $\mathrm{N}<\mathrm{S}<$ $\mathrm{E}=\mathrm{W}<\mathrm{NE}=\mathrm{NW}<\mathrm{SE}=\mathrm{SW}$ pattern completely or partly [2] [5] [7] [8], and the advantage effect of canonical position was in accordance with the pattern of $\mathrm{F}<\mathrm{B}<$ $\mathrm{L}=\mathrm{R}<\mathrm{BL}=\mathrm{BR}<\mathrm{FL}=\mathrm{FR}$ [7] [8], and (2) mental rotation strategy and analytical inference strategy were used for cardinal direction judgments [2] [5] [9].

However, the advantage effect of right and left position may be dependent of heading. With absolute direction judgments tasks, Zhou et al found that when viewer faced to the cardinal direction, the judgment performance on left or right position was better than that on noncanonical positions, while the result was reverse for noncardinal direction [3] [10]. Fig. 2 showed illustratively the result of theses studies. 


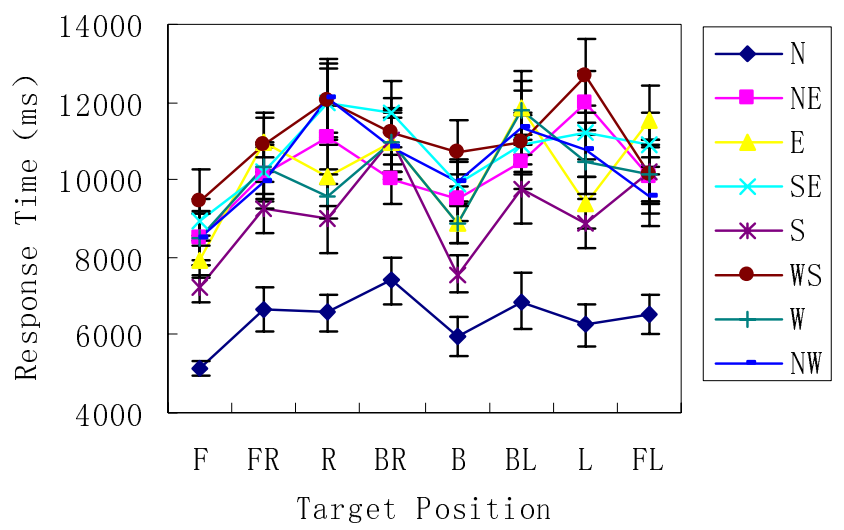

Fig. 2. Response time in absolute direction judgments was plotted as a function of target position and camera heading in one of our pervious experiments [3]

Strategies Used in Judgments. Zhou suggested that the canonical-cardinal reference advantage effect could be related to the use of different strategies in the absolute direction task [3]. With absolute direction problem shown in Fig.1, the main strategies were described as follows [2] [3] [9].

Mental rotation strategy. For the problem, the map-first mental rotation strategy can be stated as "from the map display, the current heading was rotated by 135 degree clockwise from the north, so the target lot in the 3D display should also be rotated in the same way, then the target will reach the front of the reference building, that is to say it is the north of the central object". With using this strategy, the 3D display will be looked potentially as a north-up map. In 3D-first mental rotation strategy, the statement will be described as "in order to solve the problem, the 3D display will be imagined and coordinated into the map display until the top of the 3D display reached the ground point in the southeast, and the target lot will be the top in the map after rotation, so it is the north of the central object".

Analytical inference strategy. This strategy does not involve mental rotation, "from the map, the heading faced to southeast, so the forward view of the 3D display is southeast, which is equal to say that the top or the front position in the 3D display is the southeast of the central object. So the object positioned in the bottom of the 3D display is the northwest, while the target object is near to the bottom, and the absolute direction of the target lot relative to the central object could be inferred as north." This strategy involves using the exocentric frame heading as a direction inferred cue within the egocentric frame.

In absolute direction judgments, the difference of strategies using is associated with individual and group difference [2] [4] [5] [8].

\subsection{Overview of the Study}

The purpose of this study was to investigate how strategies used in absolute direction judgments affect judgment performance, especially to test whether the advantage of 
canonical-cardinal direction effect (e.g. there is opposite performance on left or right position for cardinal direction and noncardinal direction), which found in previous studies, could be changed by strategy used in the absolute direction judgments. The basic related hypothesis is that people may use different strategies for different problem conditions. In this study, all participants were trained how to use the mapfirst mental rotation strategy and were asked to keep using the strategy to complete tasks in the whole experiment session. So if the processing pattern (e.g. north and south advantage effect, front and back advantage effect, and canonical-cardinal advantage effect) changed in this study, the basic hypothesis would be supported. The conventional measures (such as response time and accuracy) were analyzed mainly and eye movement data was also collected to supply to it.

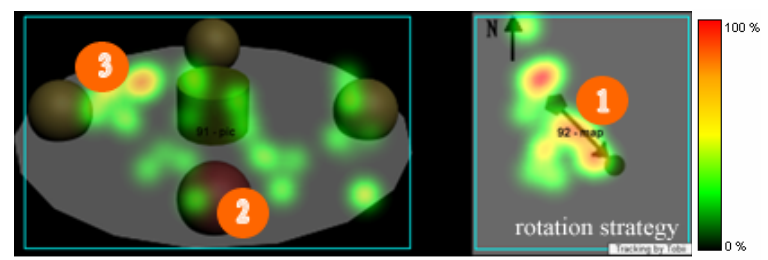

Fig. 3. Sample pattern of eyes' fixation when used map-first mental strategy to make absolute direction judgments. Areas of fixation and its duration were marked with color.

In this study we defined two areas of interest (AOI) (AOI-map display and AOI3D display) to analyze eye movement variables including durations of fixation and numbers of fixation. When using map-first mental rotation strategy, the typical pattern of eye fixation can be shown as Fig. 3. Labeled 1, 2, and 3 were used to illustrate fixation with using map-first mental rotation strategy: firstly the north was rotated 135 degree clockwise to the current heading in the map, then target object was found in the area of label 2, thirdly rotated target object 135 degree clockwise in imagination and eye fixation was transferred to the area of label 3, so the target lot was the northwest of the reference building. In the figure, the map and 3D display was defined as AOI respectively.

\section{Methods}

\subsection{Participants}

The 20 undergraduates (10 men; 10 women) from China Agricultural University, ranging in age from 18 to 22 years $(M=19.50, S D=1.10)$, participated in return for monetary compensation.

\subsection{Materials, Tasks, and Apparatus}

The tasks used in this study were similar with navigational task showed in Fig. 1. On each trial, participants first saw a test message "press the blank key to continue". When the key was pressed, the map and the 3D display were shown as Fig. 4. 
Participants were asked to identify which direction of target relative to the reference building using absolute concepts. And response was determined using the number pad on the right of the keyboard, pressing 8 for N, 9 for NE, 6 for E, 3 for SE, 2 for S, 1 for SW, 7 for W, and 7 for NW. After responding, RIGHT message with responding time (e.g., "2.567 seconds") or WRONG message without RT message was provided, and this feedback remained visible for $1.0 \mathrm{~s}$, then next trial began. A total of 8 camera headings were used, from $\mathrm{N}$ to $\mathrm{NW}$, in $45^{\circ}$ clockwise increments. For each heading, there were 8 problems with the direction of target object relative to the central object (N, NE, E, SE, S, SW, W, and NW). For each heading, the target lot would be located in canonical position (e.g. F, B, L, or R, as part a shown in Fig. 4) or noncanonical position (e.g. FL, FR, BL, or BR, as part b shown in Fig. 4) relative to the reference building from the participants' deictic view.
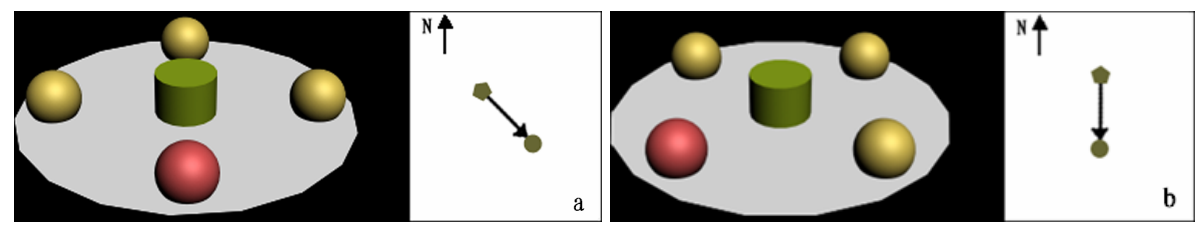

Fig. 4. Map display (exocentric reference frame) and Camera display (egocentric reference frame) used in this study. "Part a" and "part b" were designed for canonical and noncanonical position respectively. Other key information in the two parts was in accordance. In part a, the target lot was the northwest of the central building, and the target lot in part b was the northeast of the reference object.

Combined the way of computer, the instructions were presented clearly to participants by the same experimenter. Participants were instructed how to read key information on the map and camera (3D) display, and they were explained that the 3D display show the forward view from a particular camera heading and especially were trained well on how to use map-first mental rotation strategy to make response using keyboard. During the session of collecting eye movement data, participants were asked to keep appropriate posture for recording eye movement behavior. For all experiment sessions, participants were asked to answer the problems as quickly as possible without making mistakes.

The absolute direction tasks were presented on 17' monitor interfaced with personal computers. The screen resolution was set to $1024 \times 768$ pixels. Eye movements behavior were collected with a Tobii 1750 binocular remote eye tracker with $75 \mathrm{~Hz}$ temporal resolution and a 0.50 spatial resolution.

\subsection{Design}

In the north-up map, NE and NW can be rotated to $\mathrm{N}$ with the same degree, and based on the process pattern indicated in previous studies, combination of NE and NW was considered as a level of headings in this study. E-W and SE-SW were also combined as corresponding level with the same way. Similarly, the levels of target position were presented as F, FL-FR, L-R, BL-BR, and B. Since interaction effect was intended to be considered for canonical and noncanonical position respectively, response time and 
accuracy were tested with two with-in subject designs: canonical position, 5 (camera heading: N, NE-NW, E-W, and SE-SW) $\times 3$ (target position: F, R-L, and B); noncanonical position, 5 (camera heading: N, NE-NW, E-W, and SE-SW) $\times 2$ (target position: FL-FR, and BL-BR).

For eye movement data, numbers of fixation and durations of fixation were tested for camera (3D) display and map separately: for AOI-3D, target position were provided with 5 levels as F, R-L, B, FL-FR, and BL-BR; for AOI-map, 5 levels of N, NE-NW, E-W, and SE-SW were selected for camera heading.

\subsection{Procedures}

Before absolute direction judgment, participants completed 32 times practice for response using corresponding keyboard. After presenting instructions of how to respond using mental rotation strategy, 16 absolute direction judgment problems were provided for participants to practice. Then each participant completed three blocks of trials, and each block were provided $8 \times 8$ problems and presented randomly. Two blocks were completed for collecting conventional data, and the procedure was controlled by the E-prime psychological experimental software. The last block was conducted for recording eye movement behavior with eye tracker.

\section{Results}

\subsection{Conventional Behavior Measures}

Accuracy Data. Mean accuracy on absolute direction judgment is plotted as a function of heading and position in Table 1. As shown in the table: (1) the overall accuracy was the highest for $\mathrm{S}$, and response was more accurate for $\mathrm{N}$ than for other headings, and (2) response was more accurate for position of FL-FR than BL-BR. A repeated measure analysis of variance showed that the main effect of heading was significant: for canonical position, $F(4,76)=7.4, M S E=76.7, p<0.01$; for noncanonical position, $F(4,76)=7.0, M S E=73.9, p<0.01$. Pairwise comparisons supported N (98.1) = S (99.2) > E-W (94.4) = NE-NW (91.5) = SE-SW (94.7) for canonical position, and $\mathrm{S}(96.3)=\mathrm{N}(98.8)>\mathrm{E}-\mathrm{W}(91.5)=\mathrm{NE}-\mathrm{NW}(90.4)=\mathrm{SE}-\mathrm{SW}$ (91.7) for noncanonical position. The main effect of target position was significant for noncanonical position condition, $F(1,76)=7.6, M S E=65.9, p<0.5$, pairwise

Table 1. Accuracy (Percent Correct) with Standard Deviation

\begin{tabular}{lllllll}
\hline \multirow{2}{*}{ Variables } & \multicolumn{3}{c}{ Canonical Position } & & \multicolumn{2}{c}{ Non-Canonical Position } \\
\cline { 2 - 4 } \cline { 7 - 8 } & \multicolumn{1}{c}{ F } & \multicolumn{1}{c}{ L-R } & \multicolumn{1}{c}{ B } & & FL-FR & BL-BR \\
\hline $\mathrm{N}$ & $98.8 \pm 5.6$ & $96.9 \pm 6.9$ & $98.8 \pm 5.6$ & & $98.1 \pm 6.1$ & $94.4 \pm 10.3$ \\
$\mathrm{~S}$ & $100 \pm 0.0$ & $100 \pm 0.0$ & $97.5 \pm 11.2$ & & $98.7 \pm 5.6$ & $98.8 \pm 5.6$ \\
NE-NW & $93.3 \pm 13.7$ & $95.8 \pm 5.7$ & $94.7 \pm 9.8$ & & $93.3 \pm 7.9$ & $89.6 \pm 12.4$ \\
E-W & $92.5 \pm 11.4$ & $90.4 \pm 15.1$ & $91.7 \pm 12.7$ & & $92.5 \pm 8.5$ & $88.3 \pm 10.6$ \\
SE-SW & $96.7 \pm 6.8$ & $93.3 \pm 6.4$ & $94.7 \pm 11.2$ & & $93.8 \pm 8.9$ & $89.6 \pm 11.1$ \\
\hline
\end{tabular}


comparisons showed FL-FR (95.3) > BL-BR (92.1). The difference between F (96.3), L-R (95.3), and B (95.3) wasn't significant. No other main effect and interaction effect was reliable.

Response Time. Average response time for canonical position and noncanonical position is plotted of heading and position in Fig. 5. As shown in the figure, (1) as position changes from $\mathrm{F}$ to $\mathrm{B}$, the overall response time increased markedly, and response was slower for FR-FL than for BR-BL; and (2) the heading process pattern of $\mathrm{S}<\mathrm{N}<\mathrm{E}-\mathrm{W}<\mathrm{NE}-\mathrm{NW}<\mathrm{SE}-\mathrm{SW}$ was obvious.

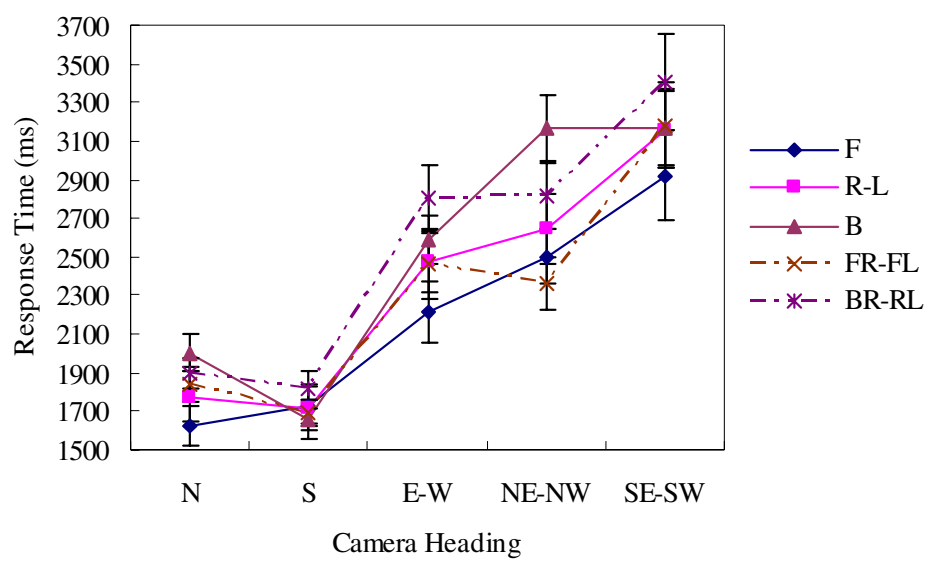

Fig. 5. Average response time for absolute direction judgments was plotted as a function of camera heading and target position, with standard error

A repeated measure analysis of variance showed the main effect of heading was significant: for canonical position, $F(4,76)=61.0, M S E=354548.1, p<0.01$; for noncanonical position, $F(4,76)=55.8, M S E=284531.2, p<0.01$. Pairwise comparisons supported N (1800.6) = S (1701.4) < E-W (2428.7) < NE-NW (2768.5) $<$ SE-SW (3084.0) for canonical position, and S (1752.4) < N (1868.5) < E-W $(2630.9)=$ NE-NW $(2585.1)<$ SE-SW (3295.1) for noncanonical position. The main effect of position was significant: for canonical position, $F(2,38)=12.0, M S E=$ 214188.4, $p<0.01$; for noncanonical position, $F(1,19)=11.3, M S E=250039.7, p<$ 0.01. Pairwise comparisons supported the statistical ordering of $F(2197.3)<\mathrm{R}-\mathrm{L}$ (2354.6) < B (2518.0) for canonical position, and FL-FR $(2307.3)<135-225$ (2545.4) for noncanonical position. Interaction effect existed in canonical position, $F(8,152)=$ 3.0, MSE $=149345.9, p<0.01$.

\subsection{Eye Movement Data}

The fixations were eliminated if the duration was less than $100 \mathrm{~ms}$. Durations and numbers of fixation are plotted of heading in AOI1 (map) and position in AOI2 (3D) in Fig. 6. 


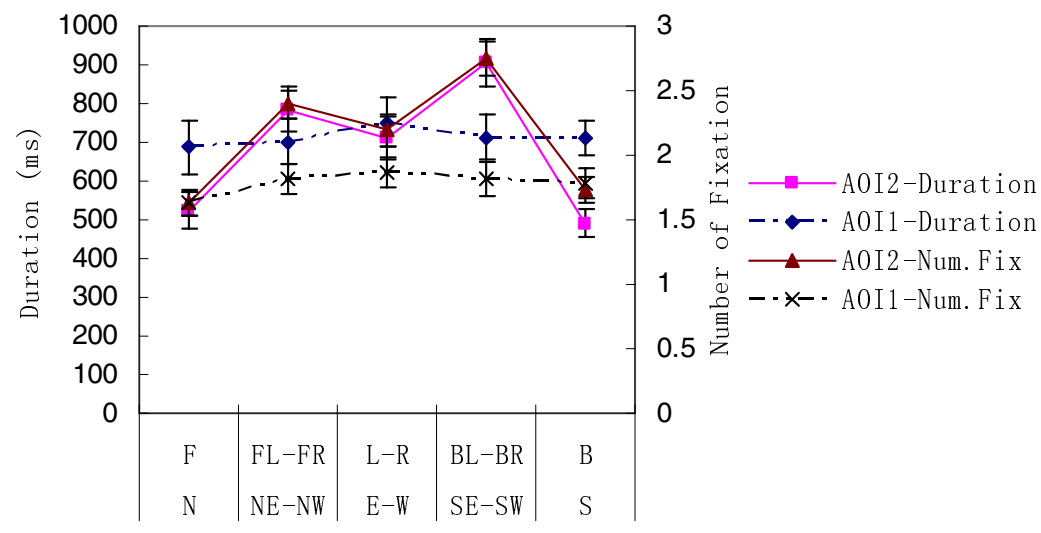

Fig. 6. Average duration and numbers of fixation were plotted as a function of camera heading and target position respectively, with standard error

For AOI1, the differences for headings were not obvious on both durations and numbers of fixation. The effect of headings was not significant with repeated measure analysis of variance: for duration, $F(4,76)=0.7, M S E=16730.5, p>0.5$; for number of fixation, $F(4,76)=2.7, M S E=0.08, p>0.5$. For AOI2, the pattern of $\mathrm{F}=$ $\mathrm{B}<\mathrm{L}-\mathrm{R}<\mathrm{FL}-\mathrm{FR}<\mathrm{BL}-\mathrm{BR}$ was evident for duration and number of duration. A repeated measures analysis of variance show that the position was significant: for duration, $F(4,76)=43.3, M S E=13283.9, p<0.001$; for number of duration, $F(4$, 76) $=39.27, M S E=0.11, p<0.001$. The overall pattern of $\mathrm{F}=\mathrm{B}<\mathrm{L}-\mathrm{R}<\mathrm{FL}-\mathrm{FR}<$ BL-BR was supported by pairwise comparison. And the comparison of AOI1 to AOI2 wasn't significant: for duration, $F(1,19)=0.001, M S E=34733.5, p<0.001$; for number of duration, $F(1,19)=13.2, M S E=0.14, p<0.001$.

\section{Discussion}

Absolute direction judgment is close related to human spatial cognitive ability, and is used in a variety of navigation related jobs such as air traffic control, driving, piloting, tracking, and police work [2] [3] [5] [9]. Previous studies were conducted to investigate how people coordinate egocentric reference frame with exocentric reference frame to make absolute direction judgments. Integrating with cardinal direction judgments, effective factors contributed to the absolute direction tasks are summarized as: (1) north and south advantage effect may be presented for headings, (2) front and back advantage effect may be formed with position of target object relative to reference object, and (3) canonical-cardinal direction advantage effect is derived from coordinating of the heading information with position information. This study was conducted to investigate how these effects varied with using map-first mental rotation strategy. No trade-off between accuracy and response time was found, so only the results of response time were discussed.

For the effect of heading, the process ordering pattern of $\mathrm{S}=\mathrm{N}<\mathrm{E}-\mathrm{W}<\mathrm{NE}-\mathrm{NW}<$ SE-SW (slow response time) was supported, and it was evident that the response was 
quicker for south than for north, and the difference was even significant in canonical position condition. Previous studies suggested that north has better performance than other headings, so in some degree the process pattern changed with the rotation strategy using. This conclusion can be supported by eye movement: it is easier to know how much degree need be rotated from north to current heading, so there was no evident difference on the durations and numbers of fixation for headings. For the effect of target position, the average response was slowest for back position in the canonical position condition. The result indicated that the back-advantage effect, which was found in previous studies [3] [5] [6] [7] [8] [10], was associated with strategies using in absolute direction judgments. However back and front positions were fixed the same duration and numbers of fixation in statistics. For interaction effect, as an example, Fig. 2 showed the advantage of L-R position for cardinal direction, and the response was detached mostly in L-R position between cardinal and noncardinal directions. The tendency wasn't affected with rotation strategy using. However, response was more centralized in back position than in other positions. The result of this study suggested that the mental rotation strategy may impact the process pattern of the headings and positions, and transformation and diversity of strategies using in absolute was proved further. But this study can not provide more details for explaining why the pattern changed with mental rotation strategy, even supplied with eye movement data (which should be used cautiously for similar navigation tasks). This can be considered in the future.

One of potential applications of these findings is the interface design of electric space. How to organize and visualize effectively information on computers has been a key issue in user interface design such as World-Wide Web and virtual environments. In order to solver the problem, spatial metaphor (e.g. data mountain, data wall, and cone tree) is well entrenched well in this field. And indeed there is need to provide both a local, egocentric reference frame and a more global, exocentric reference frame in navigational multidimensional space [1]. Based on the results of studies on cardinal direction judgments, the position of target object relative to reference object, user's forward view, and reference alignments should be considered when use spatial metaphor to improve navigation performance in electric space. However the process pattern of these factors may present difference among specific spatial presentations. This needs more investigations in future researches.

Acknowledgments. This research project was funded by a grant from the National Natural Science Foundation of China (\#30270465), and was partially supported by a grant from the China Air Force Office of Pilot Recruit.

\section{References}

1. Wickens, C.D., Hollands, J.G.: Engineering psychology and human performance (3rd). Prentice Hall, New Jersey (2000)

2. Gugerty, L., Brooks, J.: Seeing where you are heading: Integrating Environmental and egocentric reference frames in cardinal direction judgments. Journal of experimental psychology: Applied 7, 251-266 (2001) 
3. Zhou, R.: Absolute Direction Judgments Based on Integrating Egocentric and Environmental Reference Frames. Doctoral dissertation, Institute of Psychology, Chinese Academy of Sciences. Bejing (2005)

4. Gugerty, L., Brooks, J.: Reference-frame misalignment and cardinal direction judgments: Group differences and strategies. Journal of experimental psychology: Applied 10, 68-75 (2004)

5. Zhou, R., Zhang, K.: The Cardinal Direction Judgments in Integrating Environmental and Egocentric Reference Frames. Acta Psychologica Sinica 37, 298-307 (2005)

6. Zhou, R., Zhang, K.: The Direction of Integrating Reference Frames and Gender-related Difference in Cardinal Direction Judgments. Ergonomics (In Chinese) 10, 10-13 (2004)

7. Zhou, R., Yang, J., Zhang, K.: Training-related difference in Cardinal Direction Judgments Based on Integrating Reference Frames. In: Proceedings of IEA-the XVth Triennial Congress of the International Ergonomics Association, Seoul, Korea, vol. 1, pp. 1189-1192 (2003)

8. Yang, J., Zhou, R., Zhang, K.: The Training Effect and Direction Effect on Spatially Cardinal Direction Judgment. Psychological Sciences (In Chinese) 27, 1322-1325 (2004)

9. Gugerty, L., Brooks, J., Treadaway, C.: Individual differences in situation awareness for transportation tasks. In: Banbury, S., Tremblay, S. (eds.) A Cognitive Approach to Situation Awareness: Theory, Measures and Application, pp. 193-212. Ashgate Publishers, London (2004)

10. Zhou, R., Zhang, K.: Direction Judgments Based on Integrating Reference Frames in Imagination. Ergonomics (In Chinese) 11, 7-10 (2005) 